\title{
Space-Time Coding Over a Multiple-Channel Free Space Optical Link
}

\author{
Paul Keeley \\ Naval Postgraduate School \\ keeleyp1@gmail.com \\ Raymond Buettner \\ Naval Postgraduate School \\ rbuettn@nps.edu
}

\author{
Eric Stewart \\ Naval Postgraduate School \\ erstewar@nps.edu \\ David Canright \\ Naval Postgraduate School \\ dcanright@nps.edu
}

\author{
Peter Ateshian \\ Naval Postgraduate School \\ prateshi@nps.edu \\ Gurminder Singh \\ Naval Postgraduate School \\ gsingh@nps.edu
}

\begin{abstract}
Free space optical (FSO) communications in the atmosphere are characterized by additive white Gaussian noise (AWGN) and turbulent fading. We propose a combination of spatial and temporal forward error correction (FEC) coding to instantaneously correct for long-duration fades over a multiple channel link. We simulate this over a turbulent channel and identify the probability of outage of such a channel as the most significant channel parameter.
\end{abstract}

\section{Introduction}

Free space optical (FSO) communication offers the promise of tremendous bandwidth while preserving a low probability of detection and interception. Such qualities are extremely attractive for military communications applications in an increasingly congested and compromised radio-frequency (RF) spectrum. However, in addition to the additive white Gaussian noise (AWGN) typical of RF communications, FSO communications via laser also experience fluctuations in the received intensity of the signal, referred to as scintillation. These fluctuations lead to relatively long duration fades, significantly reducing the overall throughput of a FSO link.

Typical methods of overcoming fades on a single-input, single-output (SISO) system include the use of forward error correction (FEC) with large blocksizes and deep interleavers [1, 2, 3, 4]. Multiple-input, multiple-output (MIMO) systems such as those proposed by [5] and [6] typically involve spatial diversity using multiple transmitters and receivers, combined with FEC. Such systems leverage multiple paths to overcome turbulent fading, while keeping overall power constant.

More complex MIMO systems using space-time coding [7], extended Alamouti space-time coding combined with turbo codes [8], and spatial multiplexing [9], have been proposed. Additionally, some research has been done using multiple distinct channels rather than simple spatial diversity to take advantage of relay-assisted systems [10], multi-user diversity [11], or increased aggregate bandwidth [12].

This paper explores methods of increasing bandwidth by extending the sub-channel configuration and applying FEC in both the spatial and temporal dimensions. It assumes an array of $N$ receiver/transmitter pairs with tightly coupled beams-either through collimation or through techniques such as frequency diversity and physical filtering. Rather than applying fade mitigation techniques to each channel independently, we treat the output of an $N$-element transmitter as a sequence of distinct symbols. We apply FEC in the spatial dimension to provide instantaneous resilience against long-duration fades, and concatenate this with temporal coding on the source data stream to overcome the effects of AWGN. We simulate the performance of several combinations of both spatial and temporal coding to determine the effectiveness of such an arrangement over a turbulent channel in the presence of noise.

The structure of the rest of the paper follows. First, we develop the characteristics of the single-channel FSO link. This is then directly extended to characterize the multiple-channel link. Next, we provide the various coding and modulation schemes tested, and outline the channel simulator used during the testing. We then present and analyze the significant results from our simulations, and provide a few recommendations for further study in our conclusion.

\section{The Free Space Optical Channel}

In a FSO link, data is modulated onto a laser beam at the transmitter which passes through the atmosphere 
on its way to the receiver. We consider an intensity modulated/direct detection (IM/DD) system, using two levels of intensity as "on" or "off". As described in $[13,14,15]$, primary losses to the signal strength at the receiver are due to atmospheric effects such as absorption and scattering, geometric losses due to beam spreading, and variations in intensity due to atmospheric turbulence. We consider static links only which result in a fixed geometric loss, and further treat the slowly changing effects of atmospheric losses as constant for the purposes of simulation.

The current induced at the receiver is then characterized by [16] with the equation $y=s x+$ $n$, where $s$ denotes the current resulting from the intercepted signal beam, $x \in\{0,1\}$, and $n$ is AWGN. Furthermore, $s$ may be broken down as $\eta I$, where $\eta$ is the photo-current conversion ratio and $I$ is the intensity (affected by turbulence) of the normalized signal at the receiver. We do not vary the efficiency of the receiver, and will thus disregard $\eta$, leaving $s=I$. If $y$ is greater than some threshold value, it is detected as " 1 ". If not, it is detected as "0".

\subsection{Effects of Turbulent Fading}

As developed by [17], turbulence in the atmosphere results in an intensification or attenuation of the irradiance, $I$, of the received signal, which follows a double-Gamma distribution:

$p(I)=\frac{2(\alpha \beta)^{(\alpha+\beta) / 2}}{\Gamma(\alpha) \Gamma(\beta)} I^{(\alpha+\beta) / 2-1} K_{\alpha-\beta}(2 \sqrt{\alpha \beta I}), I>0$,

where $\alpha$ and $\beta$ refer to the inverses of the variances for large and small-scale turbulent cells, and $K($.$) is$ the Bessel function of the second kind. Scintillation is characterized by the Rytov variance,

$$
\chi^{2}=1.23 C_{n}^{2} k^{7 / 6} d^{11 / 6},
$$

where $C_{n}^{2}$ is the refractive index structure parameter, $\left(\mathrm{m}^{-2 / 3}\right), \quad k=2 \pi / \lambda$ is the optical wavenumber $\left(\mathrm{m}^{-1}\right)$, and $d$ is the link distance $(\mathrm{m})$. As described in [18], weak fluctuation regimes exhibit a Rytov variance between 0 and 1 , with moderate fluctuation characterized by a Rytov variance of 1 , and strong irradiance fluctuations found as the Rytov variance reaches 5 .

Returning to [17], if we assume a plane-wave approximation and further simplify the model by taking the inner scale of turbulence to be zero, we may determine $\alpha$ and $\beta$ by the following relationships:

$$
\alpha^{-1} \cong \exp \left[\frac{0.49 \chi^{2}}{\left(1+1.11 \chi^{12 / 5}\right)^{7 / 6}}\right]-1,
$$

$$
\beta^{-1} \cong \exp \left[\frac{0.51 \chi^{2}}{\left(1+0.69 \chi^{12 / 5}\right)^{7 / 6}}\right]-1 .
$$

Using $p(I)$ as in (1), for any given Rytov variance we may then calculate the probability that the received signal irradiance $I$ is below a threshold irradiance level $I_{T}$ by integrating as below:

$$
\operatorname{Pr}_{\text {fade }}=\operatorname{Pr}\left(I<I_{T}\right)=\int_{0}^{I_{T}} p_{I}(I) d I .
$$

\subsection{Effects of Noise}

Optical SNR is used throughout this paper. As in [17], to determine SNR no distinction is here made between detector shot noise due to environmental sources and the various internal sources of system noise. The Central Limit Theorem may then be invoked, allowing the total system noise to be considered additive white Gaussian noise (AWGN), with zero mean and variance $\sigma^{2}=N_{0} / 2 . \quad N_{0}$ is $k T B_{N}$, where $k$ is Boltzmann's constant, $T$ is the combined noise temperature and $B_{N}$ is the noise bandwidth. Assuming a matched filter, $B_{N}=1 / T_{b}$, where $T_{b}$ is the bitwindow (for now, considered simply the inverse of the data rate). The optical SNR may then be denoted as $s / \sigma_{N}$. The current $i$ induced by noise alone has the standard Gaussian PDF with zero mean,

$$
p_{n}(i)=\frac{1}{\sqrt{2 \pi} \sigma_{N}} \exp \left(-\frac{i^{2}}{N_{0}}\right) .
$$

By integrating this PDF from some threshold current value $i_{T}$ to infinity, the probability of a false alarm may be formulated as

$$
\operatorname{Pr}_{F A}=\int_{i_{T}}^{\infty} p_{n}(i) d i=\frac{1}{2} \operatorname{erfc}\left(\frac{i_{T}}{\sqrt{2} \sigma_{N}}\right),
$$

where $\operatorname{erfc}($.$) is the complementary error function.$

Given a normalized signal $s$, and ignoring the effects of turbulence, the output current $y=s+n$ from the matched filter has the following Gaussian PDF with mean $s$,

$$
p_{s+n}(y)=\frac{1}{\sqrt{2 \pi} \sigma_{N}} \exp \left(-\frac{(y-s)^{2}}{2 \sigma_{N}^{2}}\right) .
$$

The probability of detection is thus

$$
\operatorname{Pr}_{D}=\int_{i_{T}}^{\infty} p_{s+n}(y) d y=\frac{1}{2} \operatorname{erfc}\left(\frac{i_{T}-s}{\sqrt{2} \sigma_{N}}\right),
$$

with the probability of an instantaneous miss due to noise being simply $1-\operatorname{Pr}_{D}$. 


\subsection{Combined Effects and Extension to Multiple Channels}

The instantaneous probability of detection of a signal transmitted through a noisy, turbulent channel may be calculated as in [17] by conditioning the probability of detecting the signal plus noise over the variation in the signal induced by irradiance. However, this single $\langle\mathrm{BER}\rangle$ elides the temporal characteristic or memory of the channel and does not capture the impact of long duration fades on throughput-an impact which is exacerbated by the interaction of higher level protocols [6]. More useful is the block fading channel model used by [1], which keeps the $\operatorname{Pr}_{\text {fade }}$ constant over a time period (referred to as the temporal coherence of the channel). The channel is then modeled to be in a fade, or not. For the purposes of simulation, we took the signal value during a fade to be zero, and set $\operatorname{Pr}_{D \mid \text { fade }}=\operatorname{Pr}_{F A}$. Similarly, we took the signal value when the channel was not faded to be full strength with $\operatorname{Pr}_{D \mid \text { available }}=\operatorname{Pr}_{D}$ as in (9).

Assuming enough spatial diversity for reasonably independent, parallel paths with regards to the effects of turbulence, we model the multiple channel link as a collection of single links. While our simulation further assumes that the beam spots are not mutually interfering, for a large number of lasers, as noted by [12], the inter-channel interference produced may be combined with all other sources of noise as a single Gaussian random variable. Thus, the results we derive may be extended within reason to account for at least some level of inter-channel interference. We will discuss further methods of mitigating this in Section VI.

$\operatorname{Pr}_{\text {fade }}$ for single links may be naturally extended to a $\operatorname{Pr}$ (Outage) for a multiple-channel link. Given an $N$-laser array, a $\operatorname{Pr}_{\text {fade }}$, and some threshold number of lasers, $t$, which if simultaneously faded will render the array unusable, we calculate $\operatorname{Pr}$ (Outage) with the following equation:

$$
\operatorname{Pr}(\text { Outage })=\sum_{i=t}^{N}\left(\begin{array}{c}
N \\
i
\end{array}\right)\left(\operatorname{Pr}_{\text {fade }}\right)^{i}\left(1-\operatorname{Pr}_{\text {fade }}\right)^{(N-i)}
$$

\section{Methodology}

Given the above channel model, we now outline the coding and modulation schemes used in testing. Note that all simulations used a common optical modulation frequency of $100 \mathrm{MHz}$.

\subsection{Modulation Schemes}

A common method of modulation in the optical channel is on/off keying (OOK) [19], in which a binary 1 is transmitted by turning on the laser, and a binary 0 is transmitted by turning off the laser. In an IM/DD receiver, the presence of signal above a threshold corresponds to a binary 1 , and the absence of signal corresponds to binary 0 . This produces an obviously asymmetric channel, however, since the probability of receiving a binary 1 in error at a constant SNR is unchanging regardless of turbulence, while the probability of receiving a binary 0 in error depends on the irradiance. Clearly, in a high SNR regime, given any appreciable probability of fading, and assuming a random signal, the value 0 will be detected more frequently than 1 , leading to a decrease in entropy overall.

To address this asymmetry, binary pulse position modulation (BPPM) is a simple modulation scheme which transmits on/off pairs corresponding to binary 1 and 0 . For example, the sequence $\{0,1\}$ could be mapped to 0 and $\{1,0\}$ could be mapped to 1 . A received pair matching either $\{0,0\}$ or $\{1,1\}$ may be considered a detectable erasure. A reversed sequence (e.g., received $\{0,1\}$ when $\{1,0\}$ was sent) is thus an undetectable error. The channel may now be treated as a binary erasure channel, with symmetric erasure and error probabilities, although the bandwidth has been decreased by half.

\subsection{Codes}

Five codes are considered for use with four simulated array sizes of 8, 16, 24 and 32 lasers. All are concatenated codes using the primitive Reed Solomon $(255,32)$ code as the outer code, and a small blocksize algebraic code as the inner code, where the blocksize is matched to the array size. BPPM is used, rendering the super-channel an erasure channel and allowing recovery from a maximum of $d-1$ faded lasers, where $d$ is the minimum distance of the inner code. Occasional (up to 16 of a block of 255) failures of the inner code due to noise may be compensated for by the outer code.

Three codes use a $1 / 2$ rate inner code: the 8-bit first-order Reed-Muller(1,3) code, the 16-bit Nordstrom-Robinson code, and the 24-bit Golay code. Two additional concatenated codes were used for array sizes of 16 and 32, using the first order $\operatorname{RM}(1,4)$ and $\mathrm{RM}(1,5)$ codes respectively. Each inner code is matched to an array size, and the codewords are thus transmitted instantaneously within the symbol-window. Table 1 lists all code variations used, along with array size and threshold outage values for each code. 
As a baseline, the performance of an uncoded channel was also calculated and the performance of an $N$-sized repetition code (using OOK and maximum-likelihood decoding) was simulated for each array.

To quantify the efficiency of each code, we calculate the energy transmitted per upstream data bit $\left(E_{b}\right)$ using the equation

$$
E_{b}=\frac{1}{\text { rate }} \times T_{s} \times \frac{s^{2}}{2},
$$

where the rate refers to the coding rate, $T_{s}$ is the symbol window, and $s^{2} / 2$ is the average power received per signal bit. A lower value of $E_{b}$ indicates a more efficient code.

$\operatorname{Pr}$ (Outage) is calculated using (10). For the concatenated codes, the outage threshold is simply the minimum distance $d$ of the inner code. For the repetition codes, using maximum-likelihood decoding, the outage threshold is $N / 2$. The parameters for the five concatenated codes are displayed in Table 1.

Table 1. Code Parameters

\begin{tabular}{|c|c|c|c|}
\hline Code & $\boldsymbol{E}_{\boldsymbol{b}} / \boldsymbol{s}^{\mathbf{2}}$ & $\boldsymbol{N}$ & $\boldsymbol{t}$ \\
\hline No FEC & $0.50 \times 10^{-8}$ & All & 1 \\
\hline $\boldsymbol{R M}(1,3) / \boldsymbol{R S}(255,32)$ & $2.30 \times 10^{-8}$ & 8 & 4 \\
\hline $\boldsymbol{N R} / \boldsymbol{R S}(255,32)$ & $2.30 \times 10^{-8}$ & 16 & 6 \\
\hline $\boldsymbol{G}_{\mathbf{2 4}} / \boldsymbol{R S}(255,32)$ & $2.30 \times 10^{-8}$ & 24 & 8 \\
\hline $\boldsymbol{R M}(1,4) / \boldsymbol{R S}(255,32)$ & $3.67 \times 10^{-8}$ & 16 & 8 \\
\hline $\boldsymbol{R M}(1,5) / \boldsymbol{R S}(255,32)$ & $6.12 \times 10^{-8}$ & 32 & 16 \\
\hline
\end{tabular}

\section{Simulation Set-up}

Six combinations of turbulence conditions and $i_{T}$ were used to produce $\operatorname{Pr}_{\text {fade }}$ ranging from $<0.1 \%$ to $10 \%$. Chosen values are listed in Table 2 . A rudimentary physical layer was coded in $\mathrm{C}++$ for the simulations, handling data input, whitening, channel and line coding, and interleaving. The atmospheric channel was simulated by introducing errors into the output of the encoder. The data stream corresponding to each simulated laser was independently distorted according to the block fading model, using a Markov Chain/Monte Carlo method to apply the appropriate probabilities of fading, false alarm, and detection. Fade lengths were randomly chosen between 1 and $100 \mathrm{~ms}$ (using coherence times noted by [5]) according to a normal distribution. The calculated $\operatorname{Pr}($ Outage) for each code is graphed as a function of $\operatorname{Pr}_{\text {fade }}$ in Fig. 1 .

For each $\operatorname{Pr}_{\text {fade }}$, ten seconds of transmission time was simulated every $5 \mathrm{~dB}$ of SNR ranging from 0 to 50
$\mathrm{dB}$. This allowed 100 observations equivalent to $100 \mathrm{~ms}$ of real-time channel output at every SNR point. These observations were averaged to determine the upstream $\langle B E R\rangle$ for each code, under each set of channel and threshold conditions.

Table 2. Simulation Parameters

\begin{tabular}{|c|c|c|}
\hline$\chi^{\mathbf{2}}$ & $\boldsymbol{i}_{\boldsymbol{T}}$ & $\mathbf{P r}_{\text {fade }}$ \\
\hline 0.04 & 0.5 & 0.000675 \\
\hline 0.2 & 0.33 & 0.015002 \\
\hline 1 & 0.125 & 0.030532 \\
\hline 1 & 0.2 & 0.074877 \\
\hline 5 & 0.0625 & 0.049086 \\
\hline 5 & 0.125 & 0.10734 \\
\hline
\end{tabular}

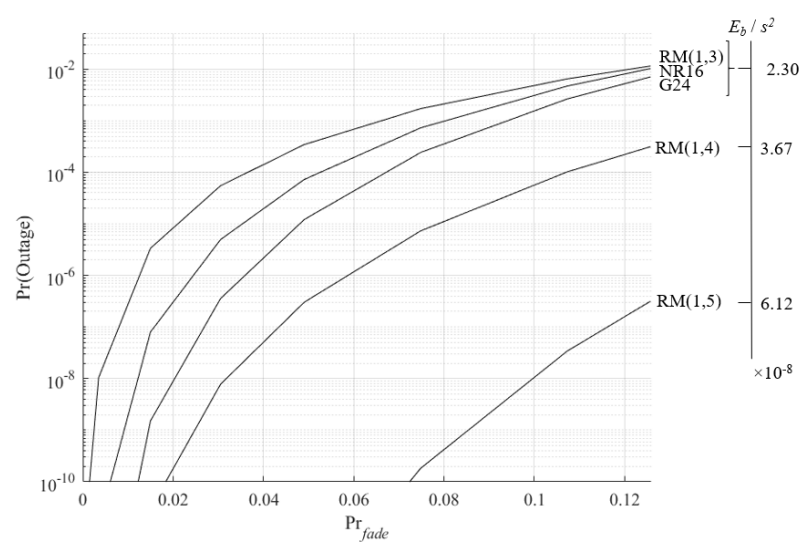

Figure 1. $\operatorname{Pr}$ (Outage) as a Function of $\operatorname{Pr}_{\text {fade }}$.

\section{Results and Analysis}

Typical outputs from the simulation are shown in Fig. 2, which depicts a 32-laser array experiencing strong turbulence as well as the calculated result from a non-coded link. Each diamond represents the $\langle\mathrm{BER}\rangle$ for an observation of $100 \mathrm{~ms}$. In all cases, the achieved $\langle\mathrm{BER}\rangle$ slowly descended as SNR increased, with a sharp decrease at the point where the FEC overpowered the noise to achieve some stable level. The link was determined to be usable if the achieved $\langle\mathrm{BER}\rangle$ in the stable portion of the plot was less than $10^{-6}$, with variability on the order of or less than $10^{-6}$. Overall, the concatenated codes based on first-order Reed-Muller codes of length 8,16 and 32 had statistically similar channel usability rates as did the repetition codes of the same length, while achieving higher throughput.

The simulated $\langle\mathrm{BER}\rangle$ in the stable regions is graphed as a function of $\operatorname{Pr}$ (Outage) in Fig. 3 . In general, simulated $\langle\mathrm{BER}\rangle$ were on the order of, 


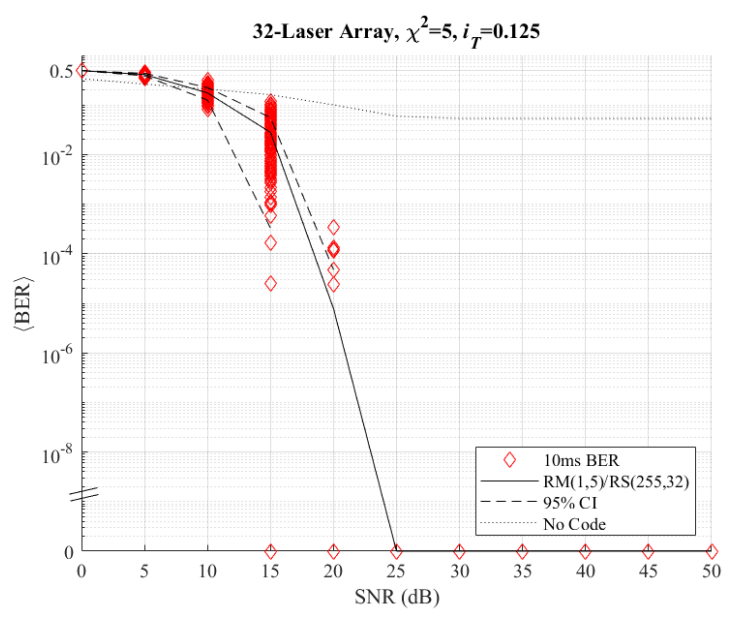

Figure 2. Typical Results, Simulated 32-Laser Array in High Turbulence.

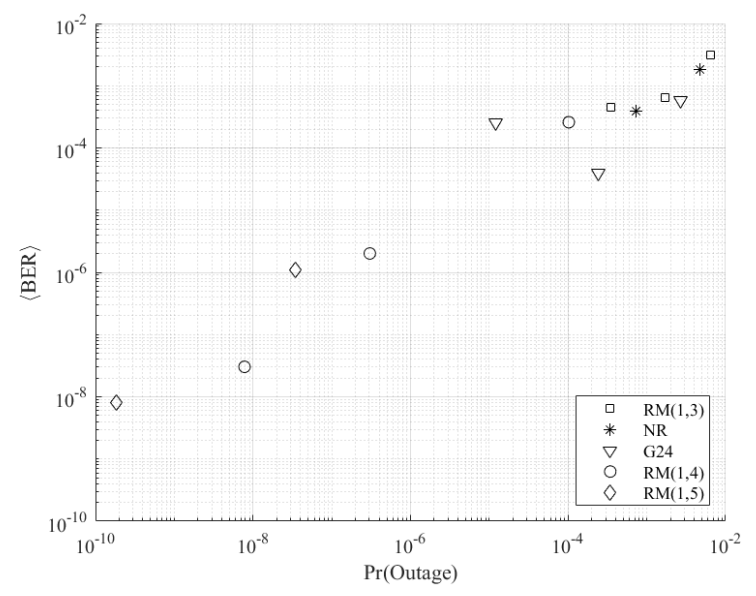

Figure 3. Simulated $\langle\mathrm{BER}\rangle$ as a function of $\operatorname{Pr}$ (Outage).

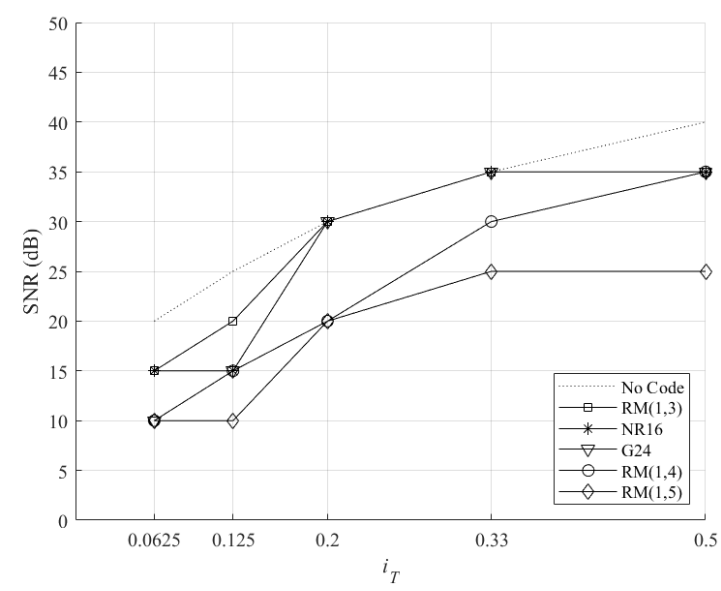

Figure 4. SNR requirement as a function of $i_{T}$. or an order of magnitude less than, the calculated $\operatorname{Pr}$ (Outage). We expect that if the simulations had been longer, eventually the $\langle\mathrm{BER}\rangle$ would have matched the $\operatorname{Pr}$ (Outage).

In each case, the usable channel appeared only after the $\operatorname{Pr}_{F A}$ had decreased to a level which depended on the $E_{b}$. This ranged from $10^{-4}$ for the three 1/2-rate codes to as high as 0.1 for the two lower-rate codes, which translated into a coding gain of 10-15 dB in terms of SNR, as seen in Fig. 4. Lowering the threshold $i_{T}$ to decrease the $\operatorname{Pr}_{\text {fade }}$ while keeping turbulence constant increased $\operatorname{Pr}_{F A}$ and typically increased the SNR requirement by approximately $5 \mathrm{~dB}$. Removing the outer (temporal) code likewise increased the SNR requirement by 5-10 $\mathrm{dB}$. However, in no case did removing the outer code render a usable channel unusable throughout the full range of SNR, indicating that the outer code was primarily correcting errors due to noise, and providing little to no fade protection.

Under our assumed resolution of individual laser spots, the use of first-order Reed-Muller codes as an inner (spatial) code in some concatenated configuration is preferred under highly turbulent conditions. The Pr(Outage) of a RM code is identical to that of a same-size spatial repetition scheme using maximum-likelihood decoding under the same channel conditions. By extension, at lower levels of $\operatorname{Pr}_{\text {fade }}$, use of higher-rate codes should become possible, enabling greater throughput.

Under suitable conditions, therefore, the use of a multiple channel link can provide an increase of several times the bandwidth of a single channel link, while providing instantaneous protection against individual fades of any length without the latency associated with typical single-channel configurations. The use of the $\operatorname{RS}(255,32)$ block code requires data inputs of 223 or more bytes, which is much less than the blocksizes required by [3] and [20], or for a deeply interleaved code as described by [6].

\section{Conclusions and Future Directions}

In this paper, we examine space-time coding using parallel transmission configurations over the MIMO atmospheric FSO channel. We identify the parameter of $\operatorname{Pr}$ (Outage) as the single most important predictor of channel performance, which over time is expected to match the $\langle\mathrm{BER}\rangle$. First-order Reed-Muller codes are seen to provide a fading protection equivalent to spatial repetition with higher efficiency than a repetition code, while higher rate arrangements decay rapidly as the $\operatorname{Pr}_{\text {fade }}$ increases.

Based on these results, we recommend the use of 
variable rate coding for future systems to allow for increased throughput when turbulent conditions permit, and increased protection from fading when conditions deteriorate. Decoding failure rates from the outer code could be used in place of dedicated pilot tones indicating a need to shift from a higher to a lower rate code.

Future directions could include use of soft-decision decoding algorithms for either the inner or outer codes. For our simulations, we used a hard-decision decoding algorithm for all codes. Recent results by [21] indicate that Reed-Muller codes achieve capacity on erasure channels using both bit and block maximum-a-posteriori (MAP) decoding. Implementation of bitwise MAP decoding could thus yield a maximum coding gain over the super-channel.

We varied only the inner code for the different concatenated variations. Other codes (e.g., convolutional, turbo or low-density parity check codes) may provide improved noise protection when used as the outer code.

As mentioned, our simulations assumed that the spot size of each laser was captured by the matched receiver. Relaxing this requirement would permit much longer distances or less tightly collimated lasers. Rather than treating the output as a series of distinct laser spots, the energy density patterns across the entire array of apertures could be used to distinguish between the $2^{N}$ possible transmitted symbols. In the case of the concatenated codes proposed, this would take advantage of the minimum distance of each inner code, reducing the number of possible outcomes. Paired with the immediate feedback found from a successful outer decoding step, it may be possible to use machine-learning algorithms to improve the response of the inner code as channel states change throughout the transmission window.

\section{Acknowledgements}

The authors wish to thank Dr. David T. Wayne and Dr. Burton H. Neuner, III of Naval Information Warfare Center, Pacific for sponsoring this research. This research was funded in part by the Naval Research Program (NPS-19-N354-A).

\section{References}

[1] S. Bourennane, P. Caussé, M. A. Khalighi, and F. Xu, "Performance of coded time-diversity free-space optical links," 24th Bienn. Symp. on Comms., pp. 146-149, 2008.

[2] L. Yang, J. Cheng, and J. F. Holzman, "Performance of convolutional coded ook im/dd systems over strong turbulence channels," 2013 International Conference on Computing, Networking and Communications (ICNC), pp. 35-39, 2013.

[3] Z. Kolka, V. Biolkova, D. Biolek, and M. Horak, "Simulation of coding for atmospheric optical channel," in 2008 Proceedings of the Mosharaka International Conference on Communications, Signals and Coding, pp. 35-40, Oct 2008.

[4] F. Davidson and Y. Koh, "Interleaved convolutional coding for the turbulent atmospheric optical communication channel," Communications, IEEE Transactions on, vol. 36, pp. 993 - 1003, 101988.

[5] E. J. Lee and V. W. S. Chan, "Part 1: optical communication over the clear turbulent atmospheric channel using diversity," IEEE Journal on Selected Areas in Communications, vol. 22, pp. 1896-1906, Nov 2004.

[6] V. W. S. Chan, "Free-space optical communications," J. Lightwave Technol., vol. 24, pp. 4750-4762, Dec 2006.

[7] E. Bayaki and R. Schober, "On space-time coding for free-space optical systems," Trans. Comm., vol. 58, pp. 58-62, Jan. 2010.

[8] V. Khare and D. Chandra, "Extended alamouti space time coding scheme with turbo coding for free space optical communication," in 2011 International Conference on Computational Intelligence and Communication Networks, pp. 359-362, Oct 2011.

[9] C. Abou-Rjeily, "Spatial multiplexing for photon-counting mimo-fso communication systems," IEEE Transactions on Wireless Communications, vol. 17, pp. 5789-5803, Sep. 2018.

[10] N. D. Chatzidiamantis, D. S. Michalopoulos, E. E. Kriezis, G. K. Karagiannidis, and R. Schober, "Relay selection protocols for relay-assisted free-space optical systems," IEEE/OSA Journal of Optical Communications and Networking, vol. 5, pp. 92-103, Jan 2013.

[11] L. Yang, X. Gao, and M.-S. Alouini, "Performance analysis of free-space optical communication systems with multiuser diversity over atmospheric turbulence channels," IEEE Photonics Journal, vol. 6, pp. 1-17, 2014.

[12] J. Akella, M. Yuksel, and S. Kalyanaraman, "Multi-channel communication in free-space optical networks for the last-mile," in 2007 15th IEEE Workshop on Local Metropolitan Area Networks, pp. 43-48, June 2007.

[13] "Prediction methods required for the design of terrestrial free-space optical links," Rec. ITU-R P.1814, International Telecommunications Union, Geneva, Switzerland, 2007.

[14] J. Parikh and V. K. Jain, "Study on statistical models of atmospheric channel for fso communication link," in 2011 Nirma University International Conference on Engineering, pp. 1-7, Dec 2011.

[15] L. B. Stotts, P. Kolodzy, A. Pike, B. Graves, D. Dougherty, and J. Douglass, "Free-space optical communications link budget estimation," Appl. Opt., vol. 49, pp. 5333-5343, Oct 2010.

[16] Jing Li and M. Uysal, "Optical wireless communications: system model, capacity and coding," in 2003 IEEE 58th Vehicular Technology Conference. VTC 2003-Fall (IEEE Cat. No.03CH37484), vol. 1, pp. 168-172 Vol.1, Oct 2003.

[17] L. C. Andrews and R. L. Phillips, Laser Beam Propagation Through Random Media. Bellingham, WA: SPIE, second ed., 2005. 
[18] R. L. P. Ammar Al-Habash, Larry C. Andrews, "Mathematical model for the irradiance probability density function of a laser beam propagating through turbulent media," Optical Engineering, vol. 40, no. 8, pp. $1554-1562-9,2001$.

[19] A. B. Carlson and P. B. Crilly, Communication Systems: An Introduction to Signals and Noise in Electrical Communication. New York, NY: McGraw-Hill, 2010.

[20] I. B. Djordjevic, B. Vasic, and M. A. Neifeld, "Multilevel coding in free-space optical mimo transmission with q-ary ppm over the atmospheric turbulence channel," IEEE Photonics Technology Letters, vol. 18, pp. 1491-1493, July 2006.

[21] S. Kudekar, S. Kumar, M. Mondelli, H. D. Pfister, E. aolu, and R. L. Urbanke, "Reedmuller codes achieve capacity on erasure channels," IEEE Transactions on Information Theory, vol. 63, pp. 4298-4316, July 2017. 